Article

\title{
Three-Dimensional LiDAR Wake Measurements in an Offshore Wind Farm and Comparison with Gaussian and AL Wake Models
}

\author{
Xin Liu ${ }^{1}$, , Lailong $\mathrm{Li}^{2}$, Shaoping Shi ${ }^{1}$, Xinming Chen ${ }^{1}$, Songhua $\mathrm{Wu}^{3}{ }^{3}$ and Wenxin Lao ${ }^{1}$ \\ 1 Huaneng Clean Energy Research Institute, Beijing 102209, China; sshi99@gmail.com (S.S.); \\ xm_chen@qny.chng.com.cn (X.C.); wx_lao@qny.chng.com.cn (W.L.) \\ 2 China Huaneng Group, Beijing 100031, China; lailong_li@chng.com.cn \\ 3 College of Marine Technology, Faculty of Information Science and Engineering, \\ Ocean University of China, Qingdao 266100, China; wush@ouc.edu.cn \\ * Correspondence: x_liu@qny.chng.com.cn
}

check for updates

Citation: Liu, X.; Li, L.; Shi, S.; Chen,

X.; Wu, S.; Lao, W. Three-Dimensional LiDAR Wake Measurements in an Offshore Wind Farm and Comparison with Gaussian and AL Wake Models. Energies 2021, 14, 8313. https:// doi.org/10.3390/en14248313

Academic Editor: Galih Bangga

Received: 1 October 2021

Accepted: 19 November 2021

Published: 10 December 2021

Publisher's Note: MDPI stays neutral with regard to jurisdictional claims in published maps and institutional affiliations.

Copyright: (c) 2021 by the authors. Licensee MDPI, Basel, Switzerland. This article is an open access article distributed under the terms and conditions of the Creative Commons Attribution (CC BY) license (https:// creativecommons.org/licenses/by/ $4.0 /)$.

\begin{abstract}
Huaneng Rudong 300 MW offshore wind farm project is located in eastern China. The wake effect is one of the major concerns for wind farm operators, as all 70 units are plotted in ranks, and the sea surface roughness is low. This paper investigated the wake intensity by combining a field test and a numerical simulation. To carry out further yaw optimization, a Gaussian wake model was adopted. Firstly, a 3D Light Detection and Ranging device (LiDAR) was used to capture the features in both horizontal and vertical directions of the wake. It indicated that Gaussian wake model can precisely predict the characteristics under time average and steady state in the wind farm. The predicted annual energy production (AEP) of the whole wind farm by the Gaussian model is compared with the calculation result of the actuator line (AL)-based LES method, and the difference between the two methods is mostly under $10 \%$.
\end{abstract}

Keywords: wind turbine; wake model; large eddy simulation (LES); LiDAR

\section{Introduction}

To increase the AEP of a wind farm, operators tend to arrange as many wind turbines as possible within a limited area. Meanwhile, wind turbines are controlled separately with the logic of maximizing the power output of every single turbine. However, many studies [1-4] have found out that when all the wind turbines are put into operation at the same time in a wind farm, the real power output is often lower than the designed best performance, and sometimes the gap is quite significant. This phenomenon is mainly caused by the aerodynamic interaction of adjacent wind turbines, or in other words, the wake effect. When wind passes through a turbine rotor, the wake effect causes a wind velocity deficit, higher turbulence intensity and larger wind shear in the downstream. The velocity deficit recovers gradually as the wake develops and dissipates entirely after a sufficiently long distance. If there are two or more wind turbines in the flow direction, the downstream wind turbines may have lower local wind velocities and less power output because of the wake effect, which will decrease the total power output for the whole wind farm. Moreover, the high turbulence intensity and wind shearing increase the dynamic load of a wind turbine, which will affect the structural performance and fatigue life of the wind turbine and increase the operating cost of the wind farm. Relevant research shows that the wake effect can account for $\sim 5-15 \%$ of the total power output loss in a typical wind farm [5]. In some extreme cases with severe wake effect, the total AEP loss of the wind farm can be $30 \%$ to $40 \%$ [6].

Wake models are mathematical models describing the wake after wind turbines, and are used to calculate the velocity distribution and the power output of adjacent wind turbines in wake areas. In the micro-siting works of wind farms, engineers need to adjust 
the distance between turbines and their layouts many times, but the results are hard to be verified via field test. The challenge becomes the motivation of developing various numerical wake models to facilitate wind farm optimization work. In early studies, with ideal conditions and the inviscid hypothesis, researchers put forward some theories to calculate the aerodynamic performance of wind turbines, such as the park model and the eddy viscosity model [7], to determine the optimum space between wind turbines. These engineering wake models provide a simple and fast way to predict the impact of turbine wake and are widely used in the wind energy industry. The simple engineering wake models make assumptions which are not always realistic and several key parameters are empirical. To produce results with higher fidelity, methods such as LES were developed based on the N-S equation, for example, the widely used actuator models [8,9]. The actuator methods simplify the physical model of the rotor while keeping its aerodynamic characteristics as much as possible. Thus, it can produce more details of the flow field than simple engineering models, but still with relatively low computational cost. With the rapid development of modern computer science and technology, the use of the computational fluid dynamics (CFD) tool has developed fast in recent decades $[10,11]$. This method can describe a more realistic physical movement of the turbine rotor, and has the highest simulation precision among all wind farm evaluation methods. However, the computational cost is still far higher than the other methods.

This study is carried out in Huaneng Rudong 300 MW offshore wind farm in eastern China, where a 3D LiDAR is used to capture the wake features of a target wind turbine, in both plan position indicator (PPI, quasi-horizontal/conical surface) and range height indicator (RHI, vertical cross section) modes. The scanning results are used to revise the Gaussian wake model. The predicted AEP of the whole wind farm by Gaussian is compared with that of AL-based LES. Further experiments on integrated offshore wake control method is also under development, by optimizing the yaw of each wind turbine in the wind farm to get the largest AEP.

\section{Research Methodology}

\subsection{Gaussian Wake Model}

The Jensen wake model [12] is widely used in commercial software like WAsP, WindPRO, Windsim, Windfarmer and OpenWind. One of the most important assumptions of the Jensen model is that the velocity of the wake flow only varies with the distance from the rotor in downstream direction, while keeping constant in the radial direction at any certain distance. However, this assumption does not agree with the field test, and may lead to an inaccurate prediction, particularly in complex terrains. Measurements have found that the velocity in the radial direction is not uniformly distributed. In fact, the real velocity deficit of wake flow shows a Gaussian distribution in a cross section away from the wind rotor, shown in Figure 1.

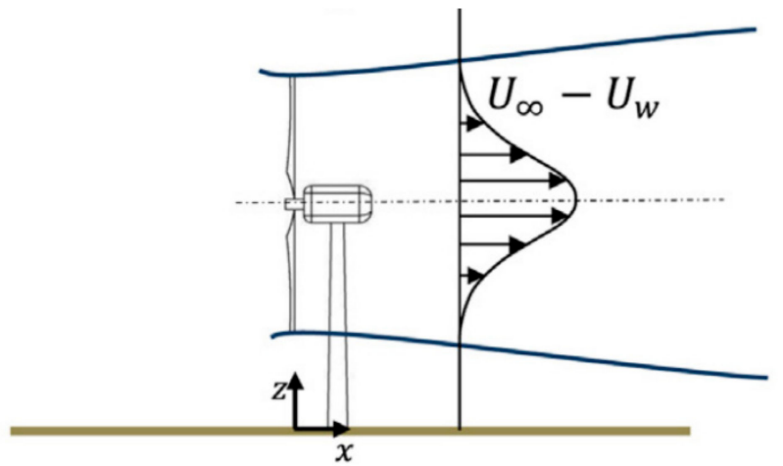

Figure 1. The velocity distribution schematic diagram in Gaussian wake model. 
In Gaussian distribution, the normal curve looks like a bell, with one high peak in the middle and lower slopes at two sides. The Gaussian curve indicates that if a random variable $X$ follows a probability distribution, where $\mu$ is the positional parameter and $\sigma$ is the scale parameter. The probability density function is:

$$
f(X)=\frac{1}{\sqrt{2 \pi} \sigma} \exp \left[-\frac{(X-\mu)^{2}}{2 \sigma^{2}}\right]
$$

Gaussian distribution has the following characteristics: (1) the peak of the normal curve is always right in the middle; (2) the normal curve is symmetrical with a center of mean value, and the curve will never intersect with the horizontal axis; (3) starting from the mean value, the normal curve decreases gradually as it goes to the left and right sides; and (4) the area between the curve and horizontal axis equals to 1 , which means the integral of the probability density function from negative infinity to positive function is 1.

The shape of the Gaussian distribution is quite similar to that of the velocity deficit in the wake area [13]. Hence, the adoption of Gaussian distribution to describe the velocity in wake flows is proper and effective.

The wake flow field has the following assumptions:

(1) The initial radius of the wake equals the rotor radius;

(2) The velocity distribution in the wake area is nonlinear;

(3) The velocity at any specific $x$ distance in the downstream has a Gaussian distribution.

A three-dimensional coordinate system is established, the y direction represents the horizontal direction of the wake cross-section, the $z$ direction represents the vertical direction of the wake cross-section.

The wake model, shown in Figure 1, also assumes a steady flow condition and no viscosity shear force, where $z$ is the height above the ground or sea level, $L$ is the downstream distance from the wind turbine, $u_{\infty}$ is the velocity of free flow, $u_{w}$ is the velocity in the wake flow, and $u_{\infty}-u_{w}$ represents the velocity deficit, marked as $\Delta u$ in the following part of the paper. $r_{0}$ is the radius of the rotor and $r$ is the radius of the wake flow at the distance of $x$ down the stream.

According to references [14-16], the complete Gaussian model for predicting the velocity distribution in the far wake region of a yawed wind turbine can be described as follows:

$$
\frac{\Delta u}{u_{\infty}}=\left(1-\sqrt{1-C_{T} \cdot \frac{\cos \gamma}{8 *\left(\frac{\sigma_{y} \sigma_{z}}{d^{2}}\right)}}\right) \cdot e^{-0.5\left((y-\delta) \sigma_{y}\right)^{2}} \cdot e^{-0.5\left(\left(z-z_{h}\right) / \sigma_{z}\right)^{2}}
$$

The widths of the wake in spanwise and the radial direction are:

$$
\begin{aligned}
& \frac{\sigma_{y}}{d}=k_{y} \frac{\left(x-x_{0}\right)}{d}+\frac{\cos \gamma}{\sqrt{8}} \\
& \frac{\sigma_{z}}{d}=k_{z} \frac{\left(x-x_{0}\right)}{d}+\frac{1}{\sqrt{8}}
\end{aligned}
$$

The beginning of the far wake region is defined as:

$$
\frac{x_{0}}{d}=\frac{\cos \gamma\left(1+\sqrt{1-C_{T}}\right)}{\sqrt{2}\left(\alpha I+\beta\left(1-\sqrt{1-C_{T}}\right)\right)}
$$

where $I$ is the turbulence intensity of the free flow at hub height, $\alpha$ and $\beta$ are constants. The deflection $\delta$ of the wake flow is the offset distance from the centerline of rotor, determined by following function.

when $x \leq x_{0}$,

$$
\frac{\delta}{d}=\theta_{c 0} \frac{x}{d}
$$


when $x>x_{0}$,

$$
\begin{gathered}
\frac{\delta}{d}=\theta_{c 0} \frac{x_{0}}{d}+\frac{\theta_{c 0}}{14.7} \sqrt{\frac{\cos \gamma}{k_{y} k_{z} C_{T}}}\left(2.9+1.3 \sqrt{1-C_{T}}-C_{T}\right) \\
\times \ln \left[\frac{\left(1.6+\sqrt{C_{T}}\right)\left(1.6 \sqrt{\frac{8 \sigma_{y} \sigma_{z}}{d^{2} \cos \gamma}}-\sqrt{C_{T}}\right)}{\left(1.6-\sqrt{C_{T}}\right)\left(1.6 \sqrt{\frac{8 \sigma_{y} \sigma_{z}}{d^{2} \cos \gamma}}+\sqrt{C_{T}}\right)}\right]
\end{gathered}
$$

if $x$ approaches to infinity, the deflection distance of the wake also approaches to a constant value.

$$
\begin{gathered}
\frac{\delta_{\infty}}{d}=\theta_{c 0} \frac{x_{0}}{d}+\frac{\theta_{c 0}}{14.7} \sqrt{\frac{\cos \gamma}{k_{y} k_{z} C_{T}}} \\
\times\left(2.9+1.3 \sqrt{1-C_{T}}-C_{T}\right) \ln \left(\frac{1.6+\sqrt{C_{T}}}{1.6-\sqrt{C_{T}}}\right)
\end{gathered}
$$

\subsection{The Actuator Line Methods}

The actuator methods are developed from blade element model (BEM), and the main difference between these two is that BEM uses cascade theories and the law of momentum, while the actuator models combine blade theories with Navier-Stokes equations (the N-S equation) [17]. There are three types of actuator models, namely the actuator disk (AD), the actuator line (AL) and the actuator surface (AS) model. The three methods differ in the way to deal with the aerodynamic force of the blades, where ADM places the aerodynamic force evenly along the circumferential direction of the rotor [18], while ALM places the force along a certain spanwise line [19], and ASM counts the aerodynamic force as a surface force [20].

The theoretical basis of the actuator method is the blade element theory, proposed by Richard Froude in 1889 [21]. The theory decomposed the complicated three-dimensional blade flow into small blade elements and analyses the force and function exchange for the flow around each blade. The blade element is a basic unit of the rotor at the any radius $r$, which is formed by the extension of a small section thickness $d_{r}$ at the specific radius $r$.

Assuming that the blade element has a chord length of $c$, the attack angle is $\alpha$, and the velocity at the location of the airfoil interface is $U_{\text {rel }}$, then the aerodynamic lift and resistance can be obtained by:

$$
\begin{aligned}
& d F_{L}=N_{b} \cdot c_{L} \cdot \frac{1}{2} \rho U_{r e l}^{2} c d r \\
& d F_{D}=N_{b} \cdot c_{D} \cdot \frac{1}{2} \rho U_{r e l}^{2} c d r
\end{aligned}
$$

where $N_{b}$ is the number of blades in a wind turbine (for most turbines, $N_{b}=3$ ). The lift coefficient $C_{L}$ and resistance coefficient $C_{D}$ is the function of attack angle $\alpha$ and Reynolds number Re.

$F_{L}$ and $F_{D}$ are decomposed along both axial and tangential directions of the blade element:

$$
\begin{aligned}
& d F_{N}=d F_{L} \cos \varphi+d F_{D} \sin \varphi \\
& d F_{T}=d F_{L} \sin \varphi-d F_{D} \cos \varphi
\end{aligned}
$$

where the tangential force $F_{T}$ pushes the rotor to rotate and to generate electricity, while the axial force $F_{N}$ produces axial thrust force to the turbine rotor.

The force $\mathbf{f}$ on the cross section of the blade, which is the function of velocity, local attack angle, the corresponding lift coefficient $C_{\mathrm{L}}$ and resistance coefficient $C_{\mathrm{D}}$, is calculated as follows:

$$
\mathbf{f}=\frac{1}{2} \rho U_{\mathrm{rel}}^{2} c \mathrm{~N}_{b}\left(C_{\mathrm{L}} e_{\mathrm{L}}, C_{\mathrm{D}} e_{\mathrm{D}}\right)
$$

where $e_{\mathrm{L}}$ and $e_{\mathrm{D}}$ are unit vectors in the direction of lift and resistance. 
The local attack angle is determined by the local inflow angle, the twist angle of the blade and the pitch angle. $U_{\text {rel }}$ is composed by the absolute velocity in axial and tangential directions:

$$
U_{\text {rel }}=\left(U_{\text {axial }}, r \cdot \Omega-U_{\theta}\right)
$$

where $\Omega$ is the rotating speed of rotor which is determined by the velocity of inflow.

The cylindrical coordinate $d A=r d r d \theta$ is applied to one micro element [22], and the load on this unit is:

$$
\mathbf{f}^{\prime}=\frac{\mathbf{f} d r}{r d r d \theta}
$$

With these methods, the N-S equation and the continuity equation are modified and will be used in the final calculation.

$$
\begin{gathered}
\frac{\partial V}{\partial t}+V \cdot \nabla V=-\frac{1}{\rho}+v \nabla^{2} V+\frac{\mathbf{f}^{\prime}}{\rho} \\
\nabla \cdot V \stackrel{=}{=}
\end{gathered}
$$

\section{Experiment}

\subsection{Wind Farm}

The wind farm in this study is the Huaneng Rudong 300 MW offshore wind farm, which is part of the "National Offshore Wind Power Development and Construction Plan (2014-2016)". It is also the first offshore wind project developed by China Huaneng Group, the state-owned utility conglomerate and the biggest electricity-generating corporation in China.

The offshore wind farm is in the near shore of Rudong County, Jiangsu Province. The center of the wind farm is about $25 \mathrm{~km}$ from the coast. The seabed topography condition is quite complex, with deep underwater gullies. The whole area is $82 \mathrm{~km}^{2}$, with the shape of an irregular quadrilateral with a length of $10 \mathrm{~km}$ form the east to west, and a width of $8 \mathrm{~km}$ from south to north. The wind farm can be divided into two parts, the northern part and the southern, shown in Figure 2, and each part has one booster station. The project consists of 70 turbines with several different capacities (4 MW, 4.2 MW and $5 \mathrm{MW}$ ) and generates about 820 million kilowatt-hours of electricity every year. The projected construction cost of the offshore wind farm is about $16,780 \sim 17,476 \mathrm{CNY} / \mathrm{kW}$.

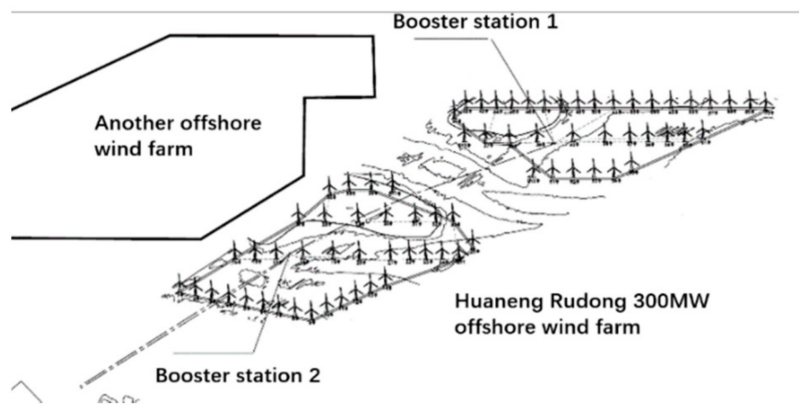

Figure 2. The layout of wind turbines.

\subsection{Experiment Setup}

To verify the accuracy of the developed Gaussian wake model, and to manage and control the wind turbines in the future, the wake distributions of $9 \#, 8 \#$ and $7 \#$ units in the Rudong offshore wind farm was measured by a 3D LiDAR, the LiDAR is shown in Figure 3. 


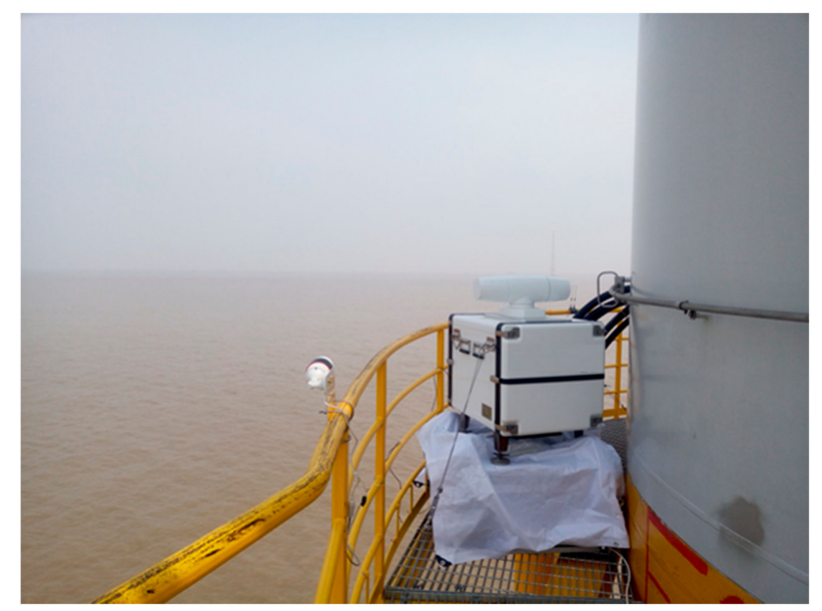

Figure 3. The photograph of the 3D LiDAR on the platform.

The work mechanism of the 3D-scanning pulsed coherent doppler LiDAR is capturing the velocities of particles in air, based on the doppler effect of laser. Compared with other measuring methods, LiDARs have the advantages of high spatial and temporal resolution, high accuracy, and high sensitivity [23-26]. Therefore, LiDARs are suitable in measuring real-time data in three dimensions of the wind farm for all weathers.

In this case, two operating modes of the pulsed coherent doppler LiDAR were carried out, which were the PPI mode (shown in Figure 4) and the RHI mode (shown in Figure 5), respectively. The PPI mode is the scanning mode where the azimuth angle is changed but the elevation angle is maintained the same. On the other hand, the RHI mode is the scanning when the azimuth angle is maintained the same but the elevation angle changes.

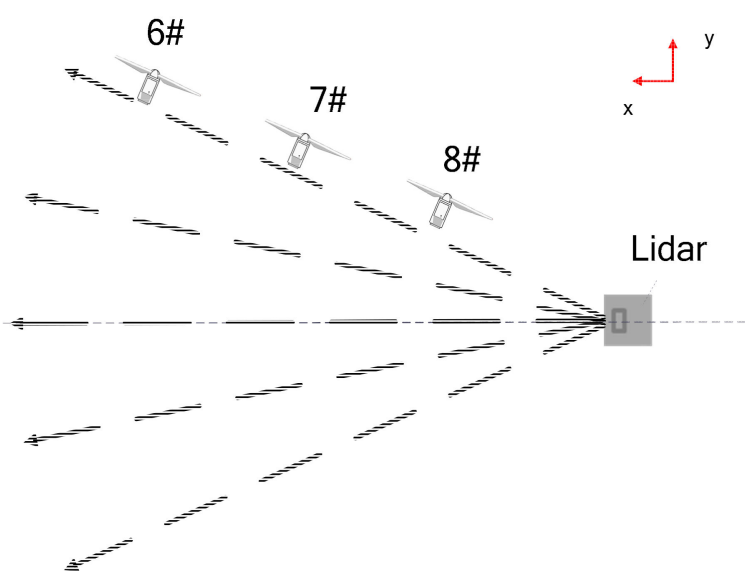

Figure 4. The schematic diagram of PPI model (the LiDAR was placed on station 9\#).

The 3D LiDAR (technical parameters shown in Table 1) is placed on the platform of 9\# wind turbine. The 8\# wind turbine (technical parameters shown in Table 2) is located at 282.7 degrees azimuth from the 3D LiDAR, and its distance from 3D LiDAR is $663.08 \mathrm{~m}$ (as shown in Figure 6). 


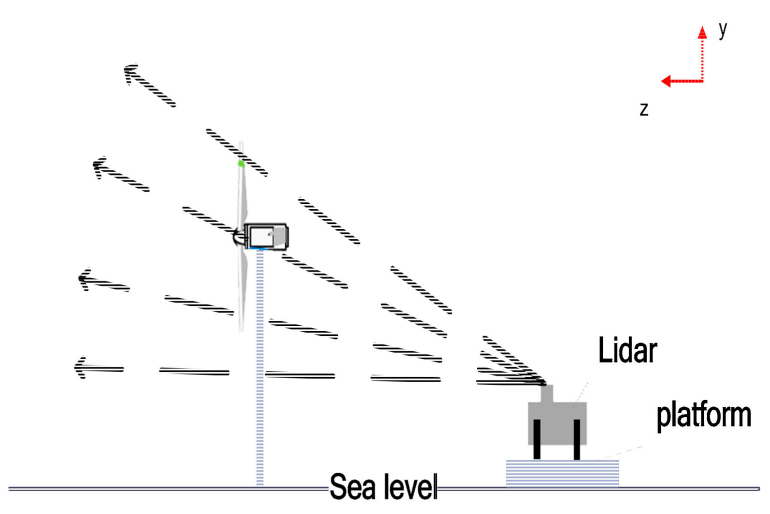

Figure 5. The schematic diagram of RHI mode.

Table 1. Main technical parameters of the 3D LiDAR.

\begin{tabular}{ccc}
\hline Index & Unit & Value \\
\hline Measuring range & $\mathrm{m}$ & $80 \sim 4000$ \\
Distance resolution & $\mathrm{m}$ & 15 (set by software) \\
Refresh rate & $\mathrm{s}$ & 1 (typical) 0.25 (fast) \\
$\leq 0.1$ (radial speed) \\
Wind speed accuracy & $\mathrm{m} / \mathrm{s}$ & $\leq 0.2$ (wind shear profile) \\
Wind direction accuracy & $\circ$ & $<3$ (Speed $>2 \mathrm{~m} / \mathrm{s}$ ) \\
Speed range & $\mathrm{m} / \mathrm{s}$ & 70 \\
Scanning model & $/$ & PPI/RHI DBS \\
Pointing precision & $\circ$ & $<0.1$ \\
Precision of servo steady & $\circ$ & $<0.1$ \\
platform (shipboard) & $\circ / \mathrm{s}$ & $1-55$ \\
Scanning speed & &
\end{tabular}

Table 2. Parameters of the wind turbine.

\begin{tabular}{ccc}
\hline Index & Unit & Value \\
\hline Rated power & $\mathrm{kW}$ & 4200 \\
IEC grade & $/$ & IEC S \\
Designed average wind speed & $\mathrm{m} / \mathrm{s}$ & 8 \\
Reference turbulence intensity & $/$ & $\mathrm{B}$ \\
Cut-in speed & $\mathrm{m} / \mathrm{s}$ & 3 \\
Rated wind speed & $\mathrm{m} / \mathrm{s}$ & 12.5 \\
Cut-out speed (10 min & $\mathrm{m} / \mathrm{s}$ & 25 \\
average) & $\mathrm{m} / \mathrm{s}$ & 30 \\
Cut-out speed & $\mathrm{m} / \mathrm{s}$ & 23 \\
Recut-in Speed $(10 \mathrm{~min}$ & & \\
average) & $\mathrm{m} / \mathrm{s}$ & 45 \\
Extreme large speed (10 min & $\mathrm{m}$ & 136 \\
average) & $\mathrm{m}$ & 14,526 \\
Rotor diameter & $\mathrm{t}$ & 99.4 \\
Swept area of rotor & $\mathrm{t}$ & 148 \\
Blade weight & &
\end{tabular}




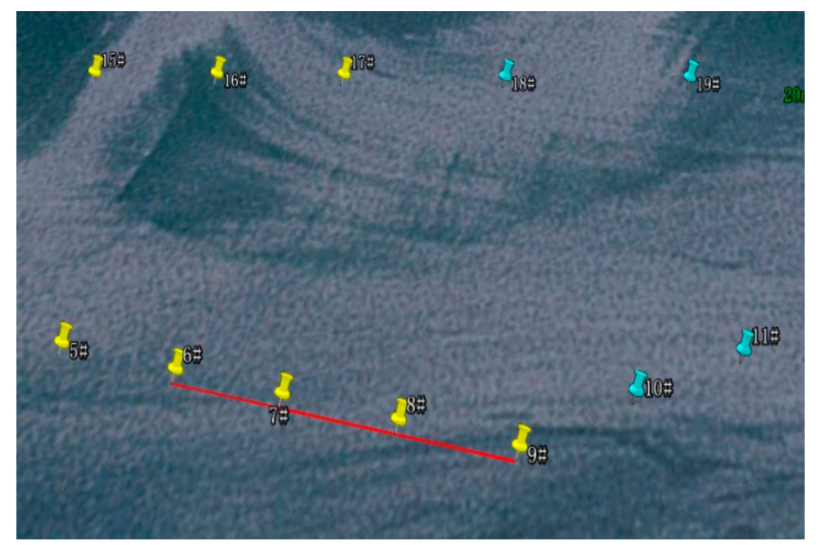

Figure 6. The schematic diagram of the measuring work in Rudong offshore wind farm (yellow pins are installed wind turbines, blue pins are planned wind turbines. The red line connects the spot of the LiDAR (at 9\#) and the spot of measured wind turbines 6\#, 7\# 8\#).

Since the height of the 3D LiDAR chassis is lower than the rail of the platform, a customized case is placed under the 3D LiDAR to ensure that the laser signal will not be obscured by the rail.

After the scanning direction of the 3D LiDAR was determined, the platform steel frame structure is connected to 3D LiDAR by basket-like screws and tightened by steel wire to ensure that the apparatus posture will not change in bad weather.

In order to detect the horizontal profile structure of the wind field at different elevations in the turbulent wake area, the PPI scanning mode is adopted. A series of elevation angles is set beforehand by programming to trace the wake accurately. To track the wake effect using the LiDAR, the LiDAR was programmed to measure the PPI mode using elevation angles at 0,3 , and 6 degrees. As the prevailing wind is in the northeast direction and the $8 \#$ turbine is located at 282.7 degrees azimuth from the 3D LiDAR, so the scanning azimuth angle is within the range of $180^{\circ}$ to $300^{\circ}$ in PPI mode. PPI scripts are set in advance according to the scanning angles.

Meanwhile, to analyze the vertical characteristic of the wake flow, the laser beam surface is perpendicular to the sea surface. The velocity vertical section at different distance is measured at the downstream of the rotor. For the 8\# turbine, the scanning elevation range is $0 \sim 18^{\circ}$, and the scanning azimuth angle is $272^{\circ}, 262^{\circ}, 252^{\circ}, 242^{\circ}$ and $232^{\circ}$ respectively. For $7 \#$ turbine, the elevation angle is set $0 \sim 9^{\circ}$, while the azimuth angles are $280^{\circ}, 275^{\circ}$, $270^{\circ}$ and $265^{\circ}$.

\subsection{Lidar Data Processing}

The velocity-azimuth display (VAD) technology is a conical scanning method to keep the elevation angle as constant and change the azimuth angle continuously in measuring the radial velocity and fitting the wind profiles, with the assumption that the horizontal wind field at any height is uniform. The PPI scanning mode uses the VAD method to inversely calculate the wind profile. When the LiDAR uses the conical scanning method to measure the horizontal wind field, a series of radial components of velocities can be obtained by changing the azimuth angle while keeping the distance from LiDAR at a constant. For further study, we treat the azimuth angle of LiDAR as an independent variable and the radial velocity component as a dependent variable, and then plot the data points in rectangular coordinate system.

The radial velocities at the same height and on the same distance circle (can also be semi-circle or sector) is a series of data following sine trend. The data can be fitted with a sinusoidal function

$$
a=A \cdot \sin (b+\varphi)
$$

where $a$ is the horizontal wind velocity, $b$ is azimuth angle. 
Take the fitting curve in Figure 7 as an example, the maximum value $a_{\max }{ }^{\prime}$ is the radial component of horizontal wind velocity. $a_{\max }$ divided by the cosine of elevation angle gives the velocity at a certain distance and the height will be obtained. The wind direction can also be read on abscissa at the point where the radial velocity has the minimum value.

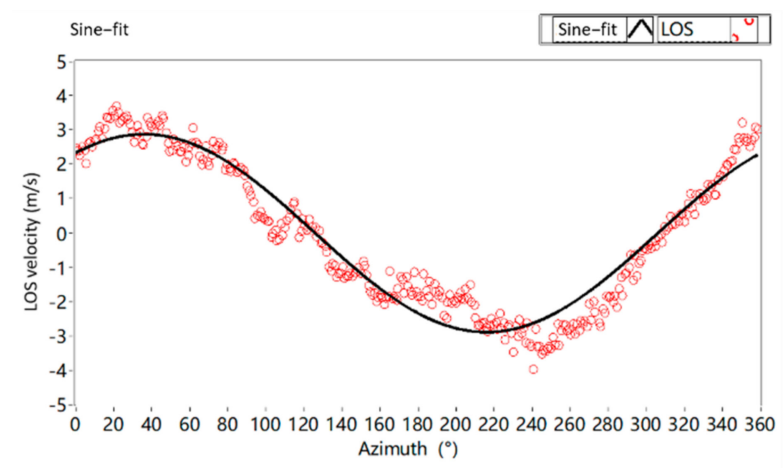

Figure 7. The fitting sine curve of radial velocity and azimuth angle.

From the analyses above, the prevailing wind direction of background wind is right along the azimuth angle where the minimum radial velocity is obtained. Based on the assumptions of VAD technology, the shape of the sine curve will change when height above sea level differs.

As the measurement of coherent doppler LiDAR is radial velocity, the real wind velocity can be calculated as follows:

$$
\begin{aligned}
u_{\text {LOS }}(\mathrm{b}, r) & =u_{\text {actual }} \cos (\theta-\varphi) \\
& =u_{\text {actual }}\left(\frac{\sqrt{r^{2}-b^{2}}}{r} \cos \varphi+\frac{b}{r} \sin \varphi\right)
\end{aligned}
$$

where $u_{\mathrm{LOS}}(\mathrm{b}, r)$ is the radial velocity, $u_{a c t u a l}$ is the real wind velocity and $\varphi$ is the wind direction.

According to former studies, the wake after turbine can fit single Gaussian model with the following equation:

$$
\begin{aligned}
u_{\text {LOS }}(\mathrm{b}, r)= & \left\{u_{a c t u a l}-\exp \left[\frac{-\left(b-b_{c}\right)}{2 s_{w}^{2}}\right]\right\} \\
& \times\left(\frac{\sqrt{r^{2}-b^{2}}}{r} \cos \varphi+\frac{b}{r} \sin \varphi\right)
\end{aligned}
$$

where $b_{c}$ is the center position of the wake flow, $s_{w}$ is the index to control the width of wake, $b_{1}$ and $b_{r}$ are used to distinguish whether the minimum value happens on the left or right side. The result is obtained by fitting method with a threshold value $p$ of 0.05 , which means when the variance is less than 0.05 , the result will not be adopted.

The aim of the analyses is to get the indexes of the wake, including the velocity deficit rate, the length and width of the wake. The velocity deficit rate is:

$$
\mathrm{VD}=\frac{u_{\text {ambient }}-u_{\text {wake }}}{u_{\text {ambient }}} \times 100 \%=\frac{u_{\infty}-u_{w}}{u_{\infty}} \times 100 \%
$$

For the single Gaussian wake, the velocity deficit rate can be simplified as:

$$
\mathrm{VD}=\frac{u_{\infty}-\left(u_{\infty}-\Delta u\right)}{u_{\infty}} \times 100 \%=\frac{\Delta u}{u_{\infty}} \times 100 \%
$$

Moreover, the width of the wake in a single Gaussian model is defined as $95 \%$ confidence interval of velocity deficit profile, that is $w=4 \sigma_{w}$. The length of the wake is the distance from the turbine to where the velocity deficit rate is decreased to $10 \%$; if the value 
does not exist, then it is defined as the distance where the deficit rate is the lowest and has a deviation of 5 to the standard $10 \%$ rate.

\section{Results}

Here we show the wake of Turbine 8 and Turbine 9 on 26 October 2017. The result of the LiDAR measurement in PPI mode is shown in Figure 8, where the LiDAR on Turbine 9 is shown in blue circle and serves as the origin in this figure. Warmer colors represent higher speed and the cooler colors have lower speed or even converse direction, the deep red or blue spots are caused by environmental noises. The small black crosses mark the wake of Turbine 9, where the speed is relatively low. Figure 9 is the result of LiDAR measurement in RHI mode, which is a vertical profile of wind field. Like PPI mode, warmer colors have higher speed and vice versa. The development of the wake is shown in yellow and green colors. Figure 10 is the predicted wake velocity of Turbine 9 with the Gaussian model. Clearly, the velocity deficit is no longer a constant value in the radial direction and follows a Gaussian law. Figure 11 shows the comparison of velocity deficit of Gaussian and LiDAR measurement. The blue dashed curve is measured data and the black curve is generated with the Gaussian model. The result shows the measured data vary up and down along the Gaussian fitted curve. By comparing to the LiDAR measurements, the Gaussian wake model could predict the trend of wind velocity deficit due to the wake's effect in the wind farm.

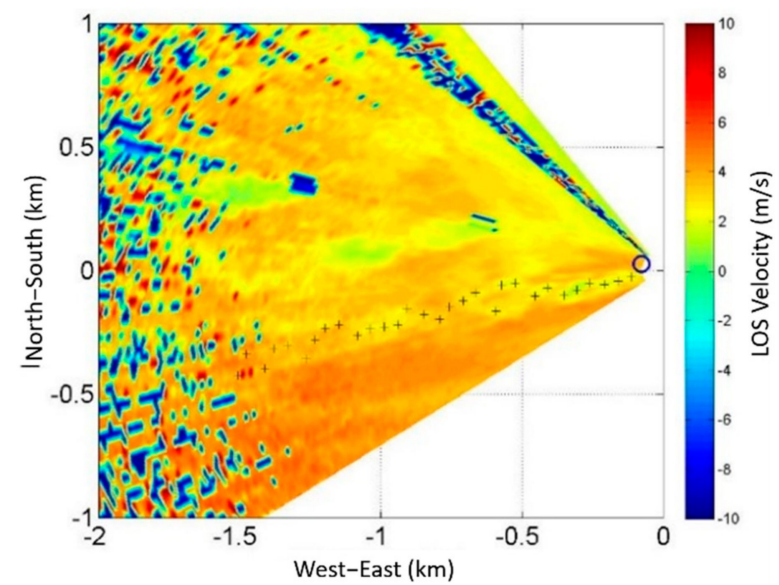

Figure 8. The visual plot of instantaneous horizontal velocities by LiDAR in PPI mode.

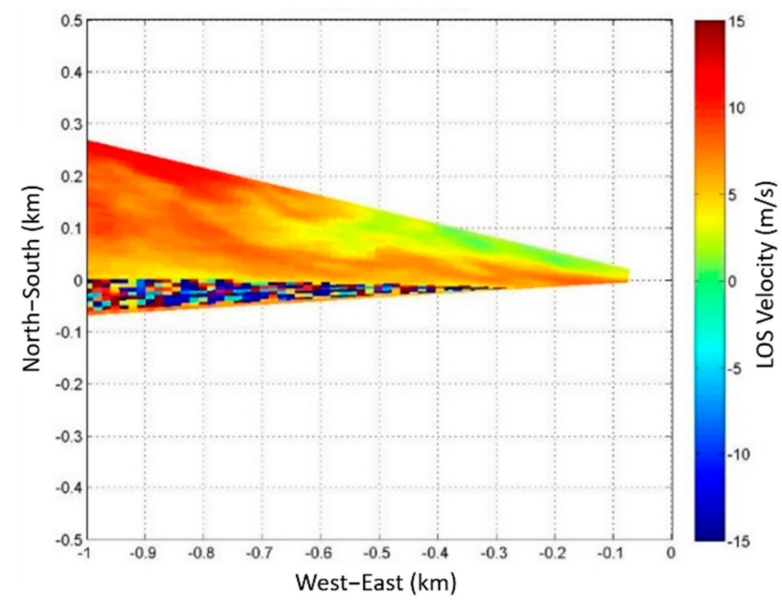

Figure 9. The visual plot of instantaneous vertical velocities by LiDAR in RHI mode. 


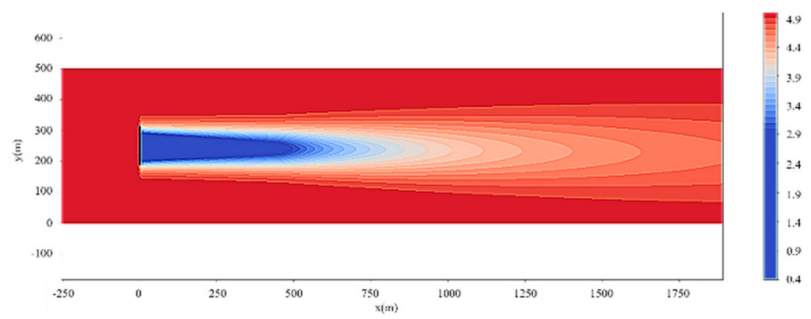

Figure 10. The predicted wake velocity distribution of 9\# with Gaussian model.

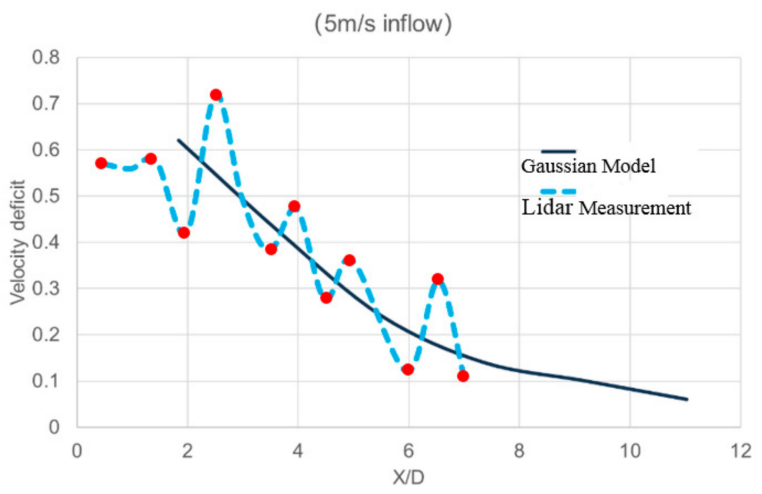

Figure 11. Comparison of velocity deficit of Gaussian and LiDAR measurement.

\section{Discussions}

Assuming that the free inflow has the direction of $135^{\circ}$, and the velocity of $12 \mathrm{~m} / \mathrm{s}$, the dynamic development of wake in Rudong offshore wind farm is obtained by the AL-LES method and calculated using the software SOWFA (developed by NREL). A total number of 30,000,000 elements were used to mesh the entire area and the transient time step was set as $0.1 \mathrm{~s}$. The result of the simulation is shown in Figure 12, where the wake development at times equal to 20 s, 120 s, $220 \mathrm{~s}$ and $820 \mathrm{~s}$ are presented. A few conclusions are made by transient LES methods:

(1) It takes some time for the wake to develop, and there is little impact on laterally area out of the wake at the beginning;

(2) The diffusion velocity of the wake equals to the velocity of background wind;

(3) In the dynamic view, the wake has volatility, and will not develop into a straight line even on flat terrain;

(4) The volatility of the wake causes the variance of power output;

(5) The average characteristics of the volatility can be approximately analyzed by steadystate wake models.

Figure 13 showed the result of the Gaussian wake model with the same assumption (the direction of $135^{\circ}$, and the velocity of $12 \mathrm{~m} / \mathrm{s}$ ). By comparing the results shown in Figures 12 and 13, the wind velocity deficit calculated by the Gaussian wake model has a quite similar trend with the wind velocity deficit calculated by the AL-based LES method.

Figures 14 and 15 is the comparison of the predicted power output of different turbines with engineering the Gaussian model and AL-based LES. The time step of AL-based LES was $0.01 \mathrm{~s}$ and the total simulation time was $20 \mathrm{~min}$. In fact, this is a huge time consumption which limits this method application in the engineering field. The first $10 \mathrm{~min}$ was dropped because in this period the flow was developing, and the units had not been in a normal stable operating condition. The power output of 36 wind turbines was predicted by the AL-based LES method and the power output of each wind turbine was averaged among a 10 min time span. Then, the power output results predicted by the Gaussian wake method and the AL-based LES method were compared. As can be observed from the figure, the difference between the calculation result of the two methods were mostly under $10 \%$. This difference is caused by the different calculation approach of the two methods. The 
Gaussian wake model method adopted a steady-state calculation approach, where each wind velocity has one corresponding power output, as for the AL-based LES method, a dynamic calculation approach is adopted, and the power output keeps changing due to the environmental condition.

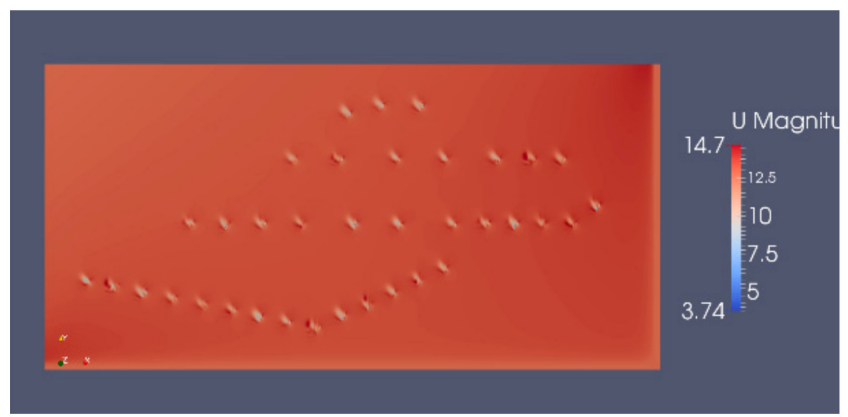

(a)

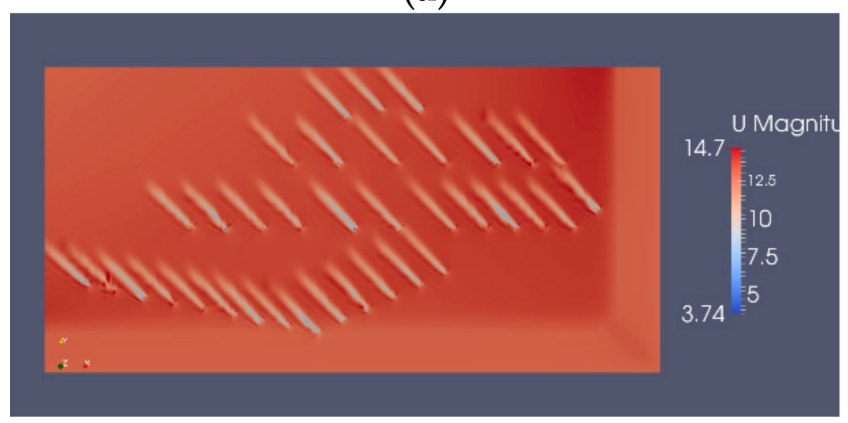

(b)

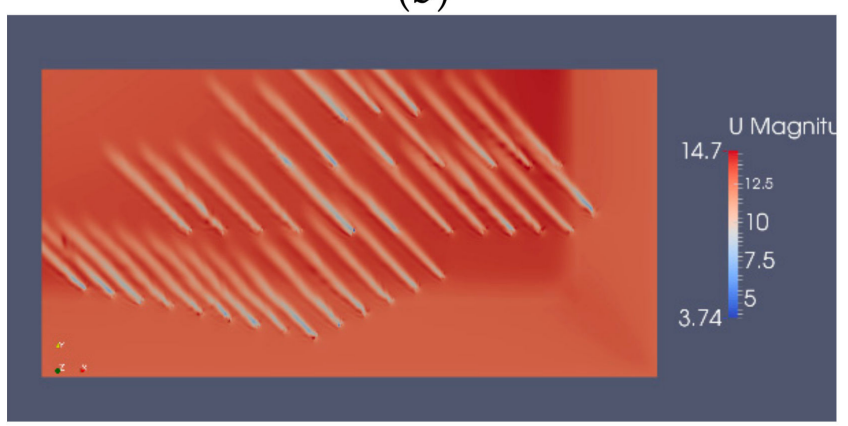

(c)

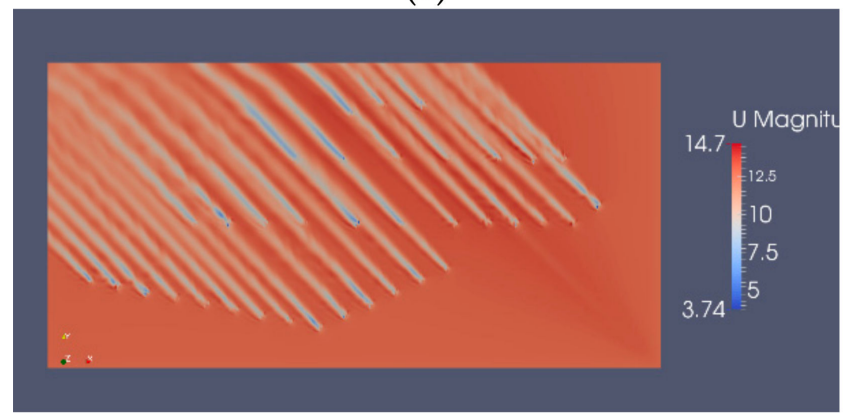

(d)

Figure 12. The distribution of wind velocity due to the wake effect of wind turbines predicted by AL-based LES method. (a) T = $20 \mathrm{~s}$, (b) T $=120 \mathrm{~s}$, (c) T $=220 \mathrm{~s},(\mathbf{d}) \mathrm{T}=820 \mathrm{~s}$. 


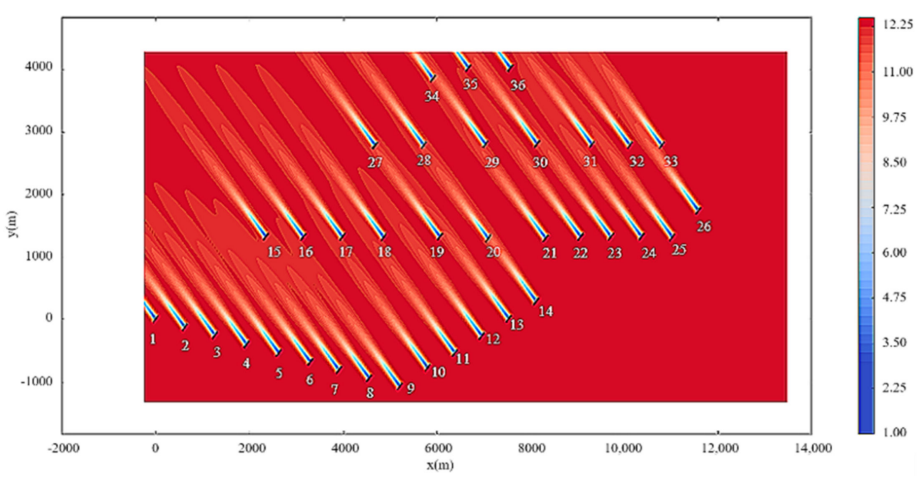

Figure 13. The wake distribution in the south part of Huaneng Rudong H12 wind farm $(v=12.5 \mathrm{~m} / \mathrm{s}$, $\operatorname{Dir}=135^{\circ}$.

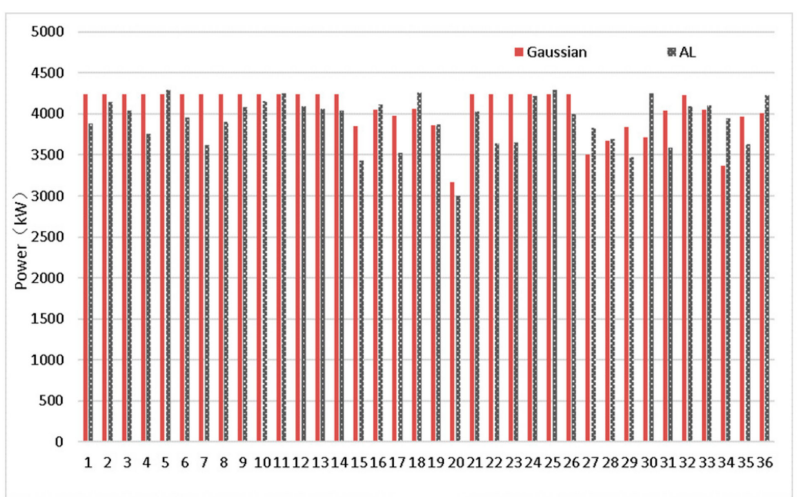

Figure 14. Comparison of predicted power output of 36 turbines in the south part with Gaussian wake model and AL-based LES.

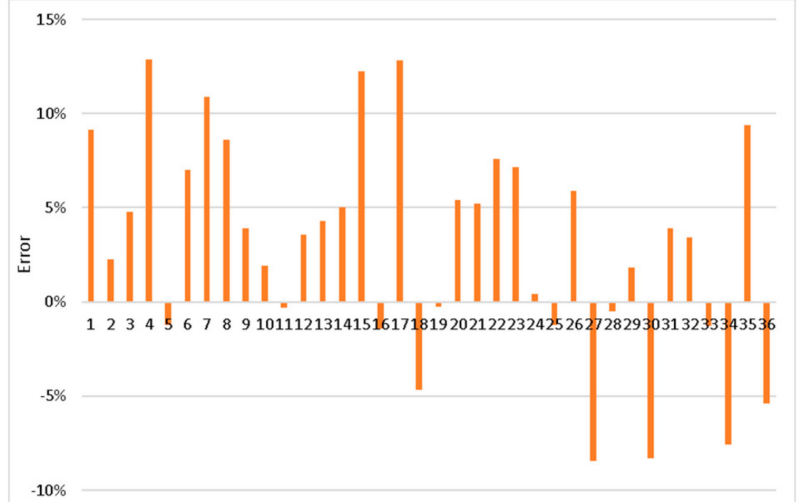

Figure 15. Comparison of power predicting errors of 36 turbines in the south part with Gaussian wake model and AL-based LES.

\section{Conclusions}

The wake effect is one of main concerns of offshore wind farm operators. Commonly, all units were arranged in ranks, and the sea surface roughness is low. This paper investigated the wake intensity by combining a field test and a numerical simulation. In order to carry out yaw optimization, the Gaussian wake model was adopted. Firstly, a 3D LiDAR was used to capture the wake features of 9\# and 8\# turbines in Huaneng Rudong 300 MW offshore wind farm in eastern China. Two scanning models, namely PPI and RHI, were adopted to obtain the horizontal and vertical information of the wake. The scanning results 
were used to revise the Gaussian wake model. The predicted AEP of the whole wind farm by the Gaussian wake model is compared with AL-based LES in the case of the direction of $135^{\circ}$ and the velocity of $12 \mathrm{~m} / \mathrm{s}$. The errors between these two methods are mostly under $10 \%$, which indicates that the Gaussian wake model can precisely predict the characteristics under time average and steady state in the wind farm. Further experimental study on the integrated offshore wake control method is also under development by optimizing the yaw of each wind turbine in the wind farm to get the largest AEP.

The Gaussian wake model will be used in the yaw optimization of Huaneng Rudong $300 \mathrm{MW}$ offshore wind farm in next stage research.

Author Contributions: Conceptualization, X.L., L.L. and S.S.; Methodology and experiment, X.L., X.C., S.W. and W.L.; Article writing, X.L., X.C. and W.L. All authors have read and agreed to the published version of the manuscript.

Funding: This work was supported by the National Key R\&D Program of China (2018YFB1501305) and China Huaneng Group Science and Technology Fund (HNKJ20-H53).

Informed Consent Statement: Not Applicable.

Conflicts of Interest: The authors declare no conflict of interest.

\section{References}

1. Bitar, E.; Seiler, P. Coordinated control of a wind turbine array for power maximization. In Proceedings of the 2013 American Control Conference, Washington, DC, USA, 17-19 June 2013; pp. 2898-2904.

2. Johnson, K.E.; Fritsch, G. Assessment of Extremum Seeking Control for Wind Farm Energy Production. Wind. Eng. 2012, 36, 701-715. [CrossRef]

3. Johnson, K.E.; Thomas, N. Wind farm control: Addressing the aerodynamic interaction among wind turbines. In Proceedings of the 2009 American Control Conference, St. Louis, MO, USA, 10-12 June 2009; pp. 2104-2109.

4. Schepers, J.G.; Van der Pijl, S.P. Improved modelling of wake aerodynamics and assessment of new farm control strategies. In Proceedings of the Science of Making Torque from Wind, Lyngby, Denmark, 28-31 August 2007; pp. 012039-012046.

5. Smith, G.; Schlez, W.; Liddell, A.; Neubert, A.; Pena, A. Advanced wake model for very closely spaced turbines. In Proceedings of the European Wind Energy Conference 2006, Athens, Greece, 27 February-2 March 2006.

6. Barber, S.; Chokani, N.; Abhari, R.S. Wind turbine performance and aerodynamics in wakes within wind farms. In Proceedings of the European Wind Energy Conference and Exhibition 2011, Brussels, Belgium, 14-17 March 2011.

7. Ainslie, J. Calculating the flowfield in the wake of wind turbines. J. Wind. Eng. Ind. Aerodyn. 1988, 27, 213-224. [CrossRef]

8. Sørensen, J.N.; Myken, A. Unsteady actuator disc model for horizontal axis wind turbines. J. Wind. Eng. Ind. Aerodyn. 1992, 39, 139-149. [CrossRef]

9. Torres, P.; Van Wingerden, J.-W.; Verhaegen, M. Modeling of the flow in wind farms for total power optimization. In Proceedings of the 2011 9th IEEE International Conference on Control and Automation (ICCA), Santiago, Chile, 19-21 December 2011; pp. 963-968.

10. Sørensen, N.; Johansen, J. UpWind: Aerodynamics and aero-elasticity Rotor aerodynamics in atmospheric shear flow. In Proceedings of the European Wind Energy Conference \& Exhibition, EWEC 2007, Milan, Italy, 7-10 May 2007.

11. Yang, X.; Sotiropoulos, F. On the predictive capabilities of LES-actuator disk model in simulating turbulence past wind turbines and farms. In Proceedings of the 2013 American Control Conference, Washington, DC, USA, 17-19 June 2013; pp. $2878-2883$.

12. Jensen, N.O. A Note on Wind Generator Interaction; Risø: Roskilde, Denmark, 1983.

13. Parada, L.; Herrera, C.; Flores, P.; Parada, V. Wind farm layout optimization using a Gaussian-based wake model. Renew. Energy 2017, 107, 531-541. [CrossRef]

14. Bastankhah, M.; Porté-Agel, F. A new analytical model for wind-turbine wakes. Renew. Energy 2014, 70, 116-123. [CrossRef]

15. Bastankhah, M.; Porté-Agel, F. Experimental and theoretical study of wind turbine wakes in yawed conditions. J. Fluid Mech. 2016, 806, 506-541. [CrossRef]

16. Niayifar, A.; Porté-Agel, F. A new analytical model for wind farm power prediction. J. Phys. Conf. Ser. 2015, 625, 012039. [CrossRef]

17. Jha, P.; Churchfield, M.; Moriarty, P. Accuracy of state-of-the-art actuator-line modeling for wind turbine wakes. In Proceedings of the 51st AIAA Aerospace Sciences Meeting including the New Horizons Forum and Aerospace Exposition, Grapevine, TX, USA, 7-10 January 2013; p. 608.

18. Hamlaoui, M.N.; Smaili, A.; Dobrev, I.; Pereira, M.; Fellouah, H.; Khelladi, S. Numerical and experimental investigations of HAWT near wake predictions using Particle Image Velocimetry and Actuator Disk Method. Energy 2022, 238, 121660. [CrossRef]

19. Nilsson, K.; Shen, W.Z.; Sørensen, J.N.; Breton, S.P.; Ivanell, S. Validation of the actuator line method using near wake measurements of the MEXICO rotor. Wind. Energy 2015, 18, 499-514. [CrossRef]

20. Kim, T.; Oh, S.; Yee, K. Improved actuator surface method for wind turbine application. Renew. Energy 2015, 76, 16-26. [CrossRef] 
21. Jin, J.; Hu, C. Stability analysis of offshore wind power monopile foundation under wind load. IOP Conf. Ser. Earth Environ. Sci. 2020, 569, 012084. [CrossRef]

22. Hansen, M. Aerodynamics of Wind Turbines, 3rd ed.; Routledge: London, UK, 2015.

23. Drechsel, S.; Mayr, G.J.; Chong, M.; Chow, F.K. Volume Scanning Strategies for 3D Wind Retrieval from Dual-Doppler Lidar Measurements. J. Atmos. Ocean. Technol. 2010, 27, 1881-1892. [CrossRef]

24. Sathe, A.; Banta, R.; Pauscher, L.; Vogstad, K.; Schlipf, D.; Wylie, S. Estimating Turbulence Statistics and Parameters from Groundand Nacelle Based Lidar Measurements IEA Wind Expert Report. DTU Wind Energy. 2015. Available online: https: / orbit.dtu. $\mathrm{dk} / \mathrm{en} /$ publications/estimating-turbulence-statistics-and-parameters-from-ground-and-n (accessed on 30 September 2021).

25. Verbeke, G.; Lesaffre, E. Power Performance Measurements of the NREL CART-2 Wind Turbine Using a Nacelle-Based Lidar Scanner. J. Atmos. Ocean. Technol. 2013, 31, 2029-2034.

26. Wagner, R.; Pedersen, T.F.; Courtney, M.; Antoniou, I.; Davoust, S.; Rivera, R.L. Power curve measurement with a nacelle mounted LiDAR. Wind. Energy 2014, 17, 1441-1453. [CrossRef] 